\title{
Analysis of Clinical Therapeutic Effect of Acupuncture on Intractable Facial Neuritis
}

\author{
E. FAN*AND Q. WEI \\ Acupuncture Center, Affiliated Hospital of Gansu University of Traditional Chinese Medicine, Lanzhou 730000, China
}

Fan et al.: Effect of Acupuncture on Intractable Facial Neuritis

\begin{abstract}
The purpose of this study is to analyze the clinical effects of acupuncture in the treatment of refractory facial neuritis. In this study, 80 patients with intractable facial neuritis are selected as the study subjects, and the patients are randomly divided into 2 groups with $\mathbf{4 0}$ people in each group. The first group is the experimental group, and the patients are treated with acupuncture and Chinese medicine. The second group is the control group, and only the same Chinese medicine treatment is given. The curative effect is determined by the scores of symptoms, signs and complications of the two groups before and after treatment. The results show that each score of the experimental group decreased more than those of the control group after treatment. This indicates that the therapeutic effect of acupuncture and traditional Chinese medicine is much better than that of the control group only treated with traditional Chinese medicine, and acupuncture has an excellent clinical therapeutic effect in the treatment of intractable facial neuritis.
\end{abstract}

Key words: Acupuncture, facial neuritis, refractory prosopoplegia

Facial neuritis, called idiopathic facial paralysis in modern medicine, also known as Bell paralysis, is a kind of disease caused by peripheral facial muscle paralysis due to non-specific inflammation of facial nerve in facial nerve tube, and then motor dysfunction of facial expression muscle group ${ }^{[1,2]}$. It is a kind of nervous system disease with high incidence, which can occur in any age group, mainly in people aged 30 to $45 \mathrm{y}$ old. The etiology and mechanism of facial neuritis have not been clearly stated ${ }^{[3]}$. With the further study and discussion of modern medicine, its etiology and pathogenesis have been further understood ${ }^{[4]}$. It is generally believed that local neurotrophic vasospasm is caused by a variety of factors such as cold and chill, circulation disorder, viral or non-specific infection, and instable autonomic nerve, resulting in facial nerve edema and swelling ${ }^{[5,6]}$. Bigoted facial paralysis is caused by prolonged non-healing of peripheral facial paralysis ${ }^{[7]}$. Patients often fail to complete their actions such as frowning, closing their eyes, cheek blowing, and showing teeth. Some patients after conventional treatment can be cured or partial recovery within 2 mo, nearly twenty percent of the patients after conventional treatment is still left joint movement (mouth and eyes move together), the phenomenon of the fault (reverse skew), facial spasm, crocodile tears and other complications. Patients with disease duration of more than 2 mo belong to the category of bigoted facial paralysis, in which more males than females. Bigoted facial paralysis, due to its complicated etiology, brings great inconvenience and mental pressure to the daily study, life and social interaction of patients. In the past, simple treatment with Traditional Chinese medicine (TCM) still led to many people who couldn't be cured quickly and developed into intractable facial neuritis ${ }^{[8]}$. Therefore, based on years of experience in treating intractable facial neuritis by attending physicians, it combines acupuncture treatment with Chinese medicine treatment in this study. Compared with the TCM treatment alone, it systematically analysis the clinical efficacy of acupuncture combined with medicine in the treatment of this disease, so as to provide a treatment method with definite curative effect, strong operability and easy to be popularized and applied in the treatment of bigoted peripheral facial paralysis. In this research, 80 patients with intractable peripheral facial paralysis who met the standards of TCM treatment in Affiliated Hospital of Gansu University of TCM from April 2014 to April 2018 are studied. According to the random principle, the patients are divided into two groups for 
observation. One group is the experimental group of 40 patients, who receive acupuncture treatment and Chinese medicine treatment; the other group is the control group of 40 patients, who only receive Chinese medicine treatment. The informed consent signed by the patients or their family members was obtained and this study was approved by the medical ethics committee of Affiliated Hospital of Gansu University of TCM.

Inclusion criteria: patients aged 19-69 y who met the diagnostic criteria; those who stopped taking other drugs and treatments for the disease during treatment; those who voluntarily participate in and agree to take this treatment; the age, course of disease, and the indicators before and after treatment of the subjects in each group are basically the same, and there is no significant difference after statistical treatment, which is comparable. Exclusion criteria: patients who don't meet the diagnostic criteria; patients under the age of 19 or over $70 \mathrm{y}$; patients with blood diseases, hematopoietic system diseases, or systemic failure caused by other diseases, as well as patients with malignant tumors and mental diseases; patients who are unable to cooperate or who have lost a case midway; patients with facial paralysis caused by trauma, facial neuroma, surgery, etc.; patients with peripheral facial paralysis caused by cerebrovascular disease, Guillain-Barre syndrome, otogenic disease, mumps, retromaxillary suppurative lymphadenitis, nerve lyme disease, posterior fossa tumor or meningitis, acoustic neuroma, cerebellopontine foot arachnoiditis, etc. Diagnostic criteria for western medicine are as follows. Medical history: patients with a course of disease greater than or equal to 2 mo who have not fully recovered after treatment by various methods; check criteria: patients with smooth lateral frontal lines, can't wrinkled forehead, frowns, increased palpebral fissure, incomplete closure, and epiphora induced by wind, the angle of the mouth is askew to the healthy side, they are inability to show teeth, shallow nasolabial groove, or impaired taste, hypersensitivity to hearing, dry eyes, and orbicularis oculi reflex, corneal reflex and blink reflex, orbicularis oris reflex are all decreased; electromyography: patients with partial facial nerve degeneration, moderate, severe or complete degeneration. Diagnostic criteria for Chinese medicine are as follows. Main symptoms: paralysis and weakness of lateral muscles, enlargement of eye fissure, incomplete closure, slanting of the mouth to the healthy side, purple or pale tongue, course of disease is more than or equal to $2 \mathrm{mo}$, and patients who have not fully recovered by various methods. Accompanied symptoms: those with qi deficiency: accompanied by short breath, lazy speech, fatigue, weak tongue, weak pulse; patients with internal heat: accompanied by red face, bad breath, dry mouth, thirst, dry stool, red tongue; patients with liver positive hyperactivity: accompanied by irritability, dizziness, red tongue, pulse string or string number; patients with weak spleen and stomach: accompanied by sallow complexion, anorexia, loose stool, and pale tongue. Acupoint selection refers to the national-level planning textbook "Acupuncture and Moxibustion" edited by Shi Xuemin and clinical experience of attending physicians. Operation method: patients are placed in supine position or sitting position and the skin of the acupoint area is disinfected with 75 $\%$ alcohol cotton balls. A disposable sterile acupuncture needle with a size of $0.30 \mathrm{~mm} \times 40 \mathrm{~mm}$ is selected for acupuncture, and the needle is retained for $30 \mathrm{~min}$ after lifting and thrusting and twisting according to the actual condition. Acupuncture is performed every $10 \mathrm{~min}$, once a day, and 30 times for a course of treatment. After 3-5 $\mathrm{d}$ of rest during the course of treatment, the second course of treatment is conducted, and the clinical efficacy, score and efficacy index are evaluated after 2 courses of treatment. Oral Chinese medicine is qu feng tong luo drink, main drugs include Angelica (15 g), Ligusticum wallichii (10 g), peach kernel (15 g), safflower (10 g), scorpion (10 g), centipede (2 PCS), stiff silkworm (10 g), and earthworm (10 g). Drugs are added or subtracted according to the specific symptoms of the patients: $15 \mathrm{~g}$ of Codonopsis pilosa and $30 \mathrm{~g}$ of Astragalus are added to the patients with qi deficiency; for patients with internal heat, $15 \mathrm{~g}$ of double blossom, $15 \mathrm{~g}$ of Forsythia, and $10 \mathrm{~g}$ of Scutellaria baicalensis are added; for the patients with liver positive hyperactivity, $15 \mathrm{~g}$ Uncaria, $10 \mathrm{~g}$ Chrysanthemum and $10 \mathrm{~g}$ Cassia are added; for patients with asthenia spleens and stomach, $15 \mathrm{~g}$ Codonopsis, $15 \mathrm{~g}$ Rhizoma Atractylodis macrocephalae and $30 \mathrm{~g}$ Poria cocos are added. One dose of medicine is decocted twice a day with water, and $300 \mathrm{~mL}$ of juice is extracted. The medicine is taken twice in the morning and evening, with a course of $30 \mathrm{~d}$. Patients are allowed to rest for 3 to $5 \mathrm{~d}$ during the course of treatment, followed by the second course of treatment, and the efficacy, score and efficacy index are evaluated after the second course of treatment. The treatment group receives acupuncture and internal administration of TCM, while the control group only receives internal administration of TCM. The method and course of TCM are the same in both groups. The symptoms are graded according to 6 symptoms, including drooping of the angle of the mouth, eardrum and cheek leakage, food retention, 
hypersensitivity to hearing, discomfort in tears, and taste disturbance. Mild symptoms are scored 1 point, obvious symptoms are scored 2 points, and severe symptoms are scored 3 points. The signs are graded according to five symptoms: frontal muscle movement, eyelid opening and closing, nasolabial groove depth, nasal shrug and platysma contractile function. Mild symptoms are scored 1 point, obvious symptoms are scored 2 points, and severe symptoms are scored 3 points. Complications are graded according to the three symptoms of joint movement, area spasm and perversion, with 1 point for minor abnormalities, 2

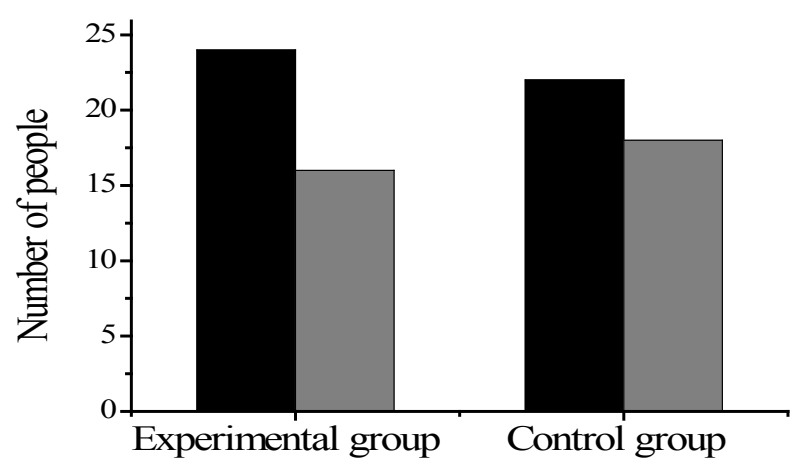

Fig. 1: Age distribution of patients with bigoted facial neuritis,

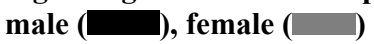

points for obvious abnormalities and 3 points for severe abnormalities. The efficacy is judged as follows. Recovery: all areas of the face are normal. Obvious effect: after careful observation, it can be observed that there is a slight weakening of the function and slight synkinetic movements, symmetry and normal tension when the face is still. The upper forehead can be moved, the eyes can be closed completely with light force and the mouth is slightly asymmetric. The face is symmetrical at rest, the tension is normal. The upper arm movement can be performed, the eye can be completely closed with light force, and the mouth is slightly asymmetrical; effective: there is obvious functional weakening, but no damaging asymmetry between the two sides, and minor synkinetic movements, contractures, and lateral spasms can be observed, the tension is normal when the face is at rest, the upper frontal movement is weak, the eye can be completely closed with light force, and the mouth is obviously asymmetric. Invalid: the face is not symmetrical when still, the upper forehead has no movement, the eyes can't be completely closed, and the mouth has slight movement. The curative effect index is to evaluate the curative effect index according to the change of the total score of symptoms and signs. Curative effect
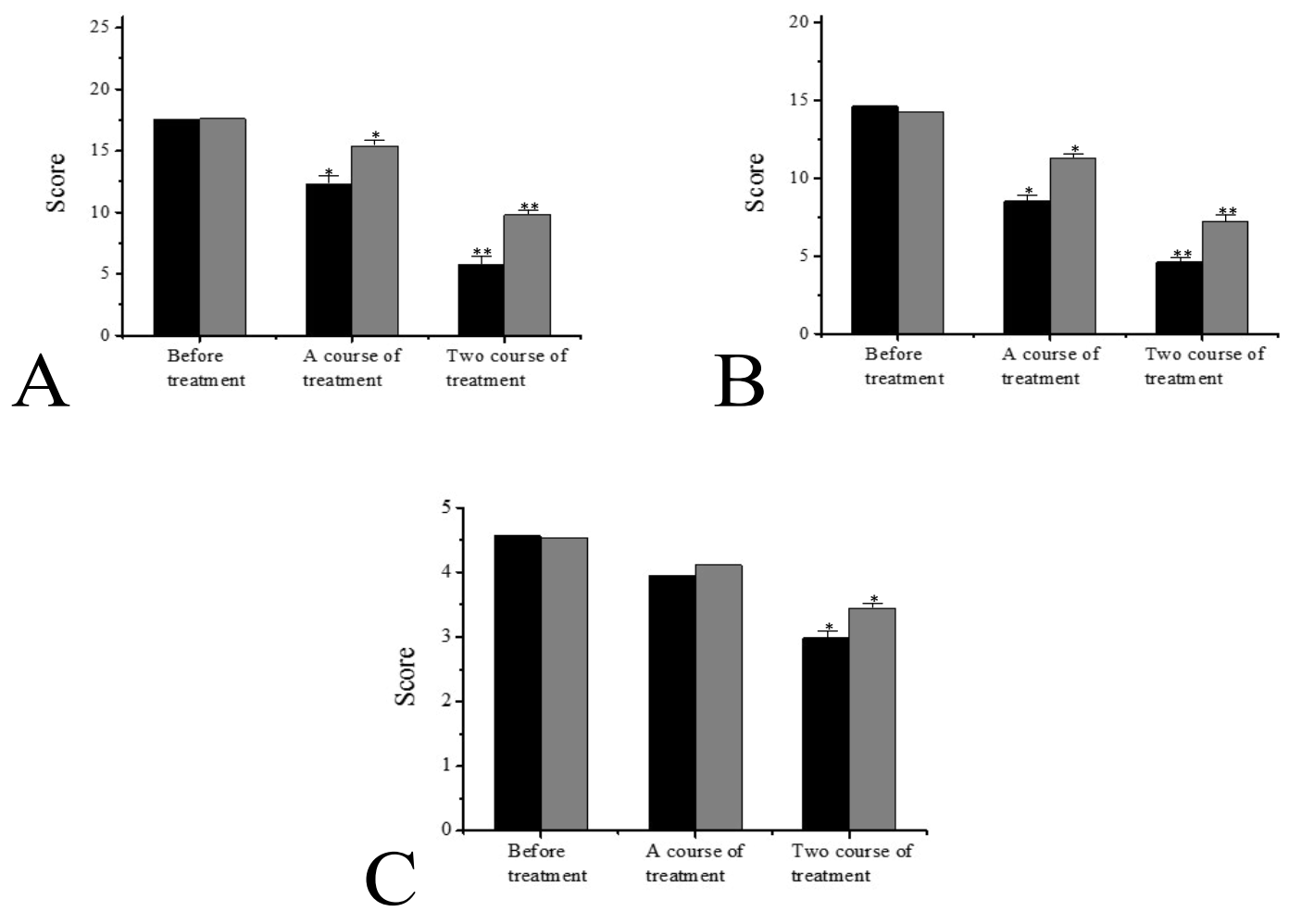

Fig. 2: Comparison of the scores between the two groups before and after treatment

A. Comparison of total scores of symptoms in the two groups; B. Comparison of the total scores of the signs of the two groups; C. Comparison of total scores of complications in the two groups. Experimental group Note: * indicates $\mathbf{p}<0.05$, and $* *$ indicates $\mathbf{p}<0.01$. 
index $=($ total score before treatment-total score after treatment $) \div$ total score before treatment $) \times 100 \%$. According to the change of the efficacy index, the merits and demerits of the efficacy index are determined. Excellent: efficacy index is greater or equal to $85 \%$; good: efficacy index is greater or equal to $66 \%$; medium: efficacy index is within $65 \%-26 \%$; poor: efficacy index is less than or equal to $25 \%$. The measurement data are expressed as mean \pm standard deviation $(\mathrm{x} \pm \mathrm{s})$, and the paired $\mathrm{t}$ test is used for comparison before and after the experiment. $\mathrm{X}^{2}$ test is used to compare the efficacy of each group in terms of the sample rate, and rank sum test is used for the ordered multiple independent samples. In addition, $p<0.05$ is considered statistically significant. A total of 80 subjects are included in this study, including 46 males $(57.5 \%)$ and 34 females $(42.5 \%)$. The average age of onset is $47.8 \mathrm{y}$ old, the minimum age is $20 \mathrm{y}$ old, the maximum age is $69 \mathrm{y}$ old, the longest course of disease is $1.2 \mathrm{y}$ and the shortest is 2 mo. There are 24 males and 16 females in the experimental group, and 22 males and 18 females in the control group, as shown in fig. 1. Comparison of total symptom scores between the two groups after treatment: fig. 2a shows that after treatment, the total score of symptoms in the two groups improved between treatment courses and before treatment. After the first course of treatment, the total score of symptoms in each group is compared, and there are significant differences between the experimental group and the control group $(p<0.05)$. After the second course of treatment, the total scores of symptoms in each group are significantly different between the experimental group and the control group $(p<0.01)$. And there were significant differences between the two groups before and after treatment. The total scores of signs of the two groups of patients after treatment are compared, after the first course of treatment, there is a significant difference between the experimental group and the control group $(p<0.05)$. The total score of symptoms and signs of each group after the second course of treatment is significantly different between the experimental group and the control group $(\mathrm{p}<0.01)$. There are significant differences between the two groups before and after treatment. And fig. $2 \mathrm{~b}$ shows that the total score of symptoms and signs in the experimental group improved well. Fig. 2c shows the comparison of complication scores between the two groups. After statistical treatment, there is no significant difference between the total score after one course of treatment and before treatment $(p>0.05)$. Compared with the total score before treatment, there are significant differences after two courses of treatment $(\mathrm{p}<0.05)$. This indicates that each group have an improvement effect on the treatment of complications, but the recovery is slow, the improvement effect is not significant after one course of treatment $(p>0.05)$, and the improvement effect is significant after two courses of treatment $(p<0.05)$. Safety evaluation: in clinical trials, no adverse effects are found on normal physiological status indicators (such as consciousness, sleep, pulse, heart rate, respiration, etc.) by the treatment methods adopted in each group, and no adverse reactions such as needle stagnation, broken needle and hematoma are found. Only individual patients have occasional needle sickness when sitting, and no more needle sickness occurs when they are in a lying position. Patients taking Chinese medicine internally occasionally have gastral cavity discomfort and slight diarrhea symptoms, which will be improved by taking them after meals or by adding or subtracting drugs as appropriate. Fig. 3 shows the change trend of the experimental group and the control group in each score before and after treatment. The scores of the experimental group decreased after acupuncture and Chinese medicine treatment, indicating that the treatment effect is better than that of the control group treated only with TCM. In the experimental group, 9 cases are cured, 15 cases are obviously effective, 12 cases are effective, 4 cases are ineffective, and the cure rate is $21.95 \%$. In the control group, 2 cases are cured, 9 cases are obviously effective, 11 cases are effective and 18 cases are ineffective effect, and the cure rate is $5.0 \%$, as shown in fig. $4 \mathrm{a}$. The cure rate of the experimental group is significantly different from the control group $(p<0.05)$, indicating that the clinical cure rate of the experimental group is better than that of the control group, and the difference was significant $(p<0.05)$. The total effective rate of the treatment group is $90.0 \%$ and that of the control group is $55 \%$, indicating that the total effective rate of the experimental group is significantly different from that of the control group $(p<0.01)$. The comparison of cure rates is shown in fig. $4 \mathrm{~b}$, and the comparison of effective rates is shown in fig. 4c. Among the 80 patients with intractable facial neuritis, males are slightly more than females, which is basically consistent with the results of most studies in recent years. However, it is not completely certain that the incidence of intractable facial neuritis in males is definitely higher than that in females, which needs to be clarified by follow-up studies with a larger sample size and more regions. The results of this study show that patients with intractable facial neuritis may be at various ages, with an average 

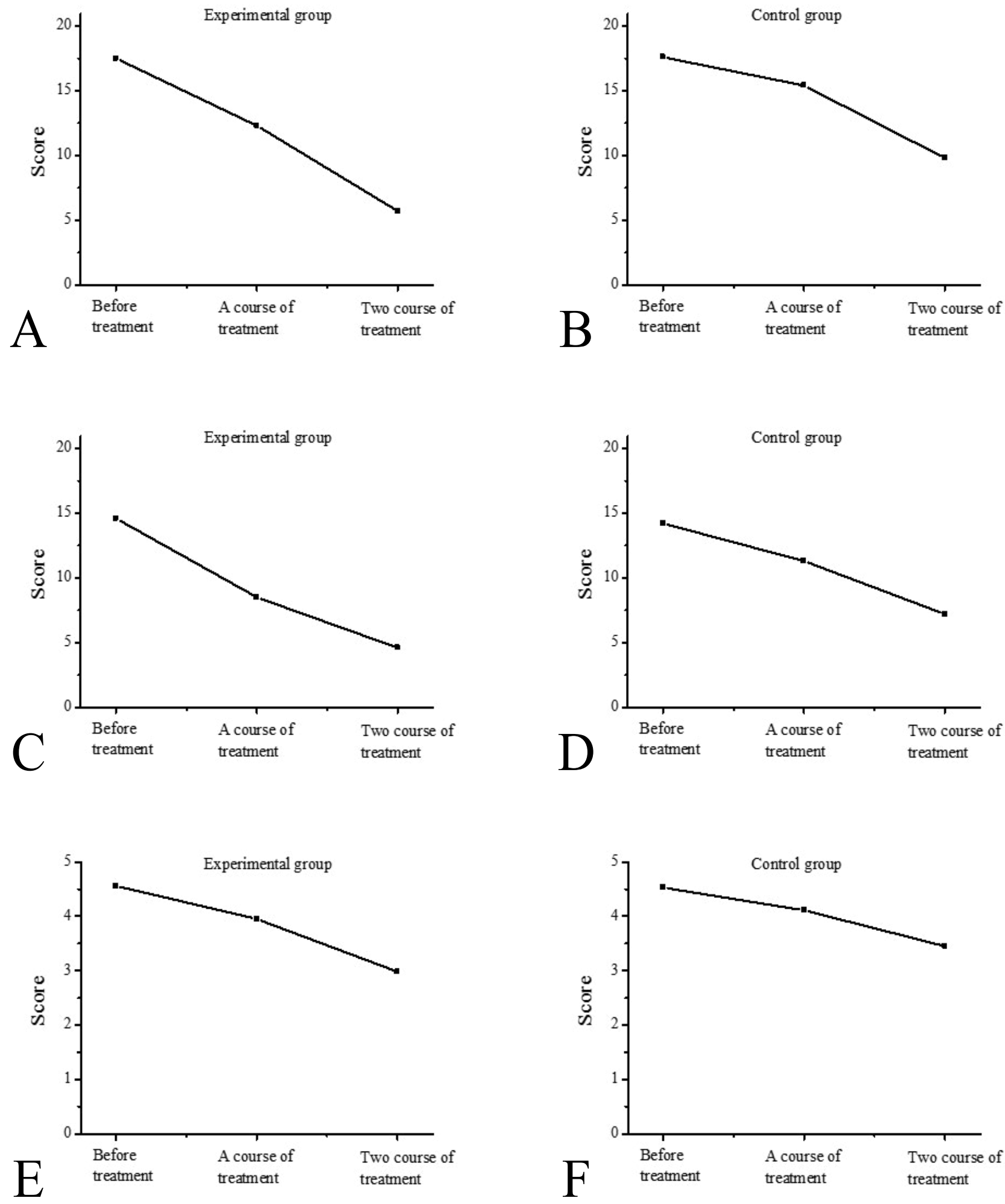

Fig. 3: The change trend of each score before and after treatment in the two groups

$A$ and $B$ : change trend of symptom score before and after treatment, $C$ and $D$ : change trend of sign score before and after treatment, $E$ and $F$ : change trend of complication score before and after treatment 

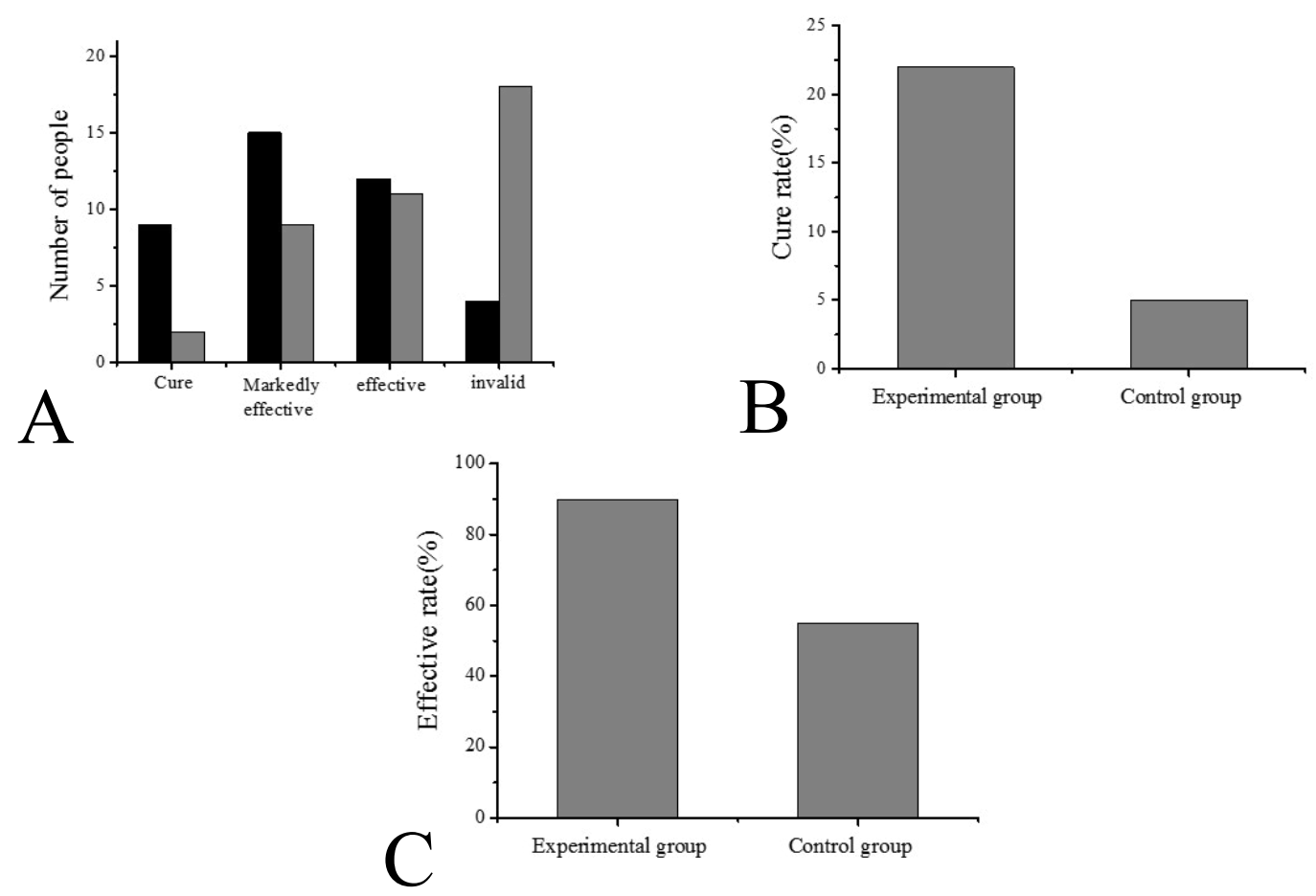

Fig. 4: Comparison of the efficacy of the two groups of patients

A: comparison of the number of patients with different curative effects between the two groups; B: comparison of cure rates between the two groups of patients; C: Comparison of effective rates between the two groups of patients, Experimental group ( $\square$, control group ( $\square$

age of $47.8 \mathrm{y}$, a minimum age of $20 \mathrm{y}$, a maximum age of $69 \mathrm{y}$, a maximum duration of $1.2 \mathrm{y}$ and a minimum duration of 2 mo. After acupuncture and TCM treatment for patients in the experimental group and the same TCM treatment for patients in the control group, it is found that the scores of patients in the three aspects of symptoms, signs and complications in both groups decreased. There is no significant difference in scores between the two groups before treatment, but the scores of patients in the experimental group after treatment are significantly lower than those in the control group. It indicates that the therapeutic effect of acupuncture and TCM is much better than the single TCM, and acupuncture has an excellent clinical therapeutic effect in the treatment of intractable facial neuritis.

\section{Acknowledgements:}

None

\section{Conflict of Interests:}

The authors declared no conflict of interest.

\section{REFERENCES}

1. Yue J, Liu M, Li J, Wang Y, Hung ES, Tong X, et al. Acupuncture for the treatment of hiccups following stroke: a systematic review and meta-analysis. Acupunct Med 2017;35(1):2-8.

2. Wang Z, Wang X, Liu J, Chen J, Liu X, Nie G, et al. Acupuncture treatment modulates the corticostriatal reward circuitry in major depressive disorder. J Psychiatr Res 2017;84:18-26.

3. Zhao L, Chen J, Li Y, Sun X, Chang X, Zheng H, et al. The long-term effect of acupuncture for migraine prophylaxis: a randomized clinical trial. JAMA Intern Med 2017;177(4):50815.

4. Wang T, Li Z, Ge T, Zhang M, Yuan A, Yang J. Summary of Professor YANG Jun's Experience for Intractable Facial Paralysis. Zhongguo Zhen Jiu 2017;37(6):649-51.

5. Xu P, Jin A, Dai B, Li R, Li Y. Surgical timing for facial paralysis after temporal bone trauma. Am J Otolaryngol 2017;38(3):269-71.

6. Eviston TJ, Chong LS, Clark JR. Reply: Validation of the clinician-graded electronic facial paralysis assessment. Plast Reconstr Surg 2018;141(4):615e-6e.

7. Vajpayee D, Mallick A, Mishra AK. Post Temporal bone fracture facial paralysis: Strategies in decision making and analysis of efficacy of surgical treatment. Indian J Otolaryngol Head Neck Surg 2018;70(4):566-71.

8. Rozen SM. Facial reanimation: Basic surgical tools and creation of an effective toolbox for treating patients with facial paralysis. Part A: functional muscle transfers in the long-term facial palsy patient. Plast Reconstr Surg 2017;139(2):469-71.

This is an open access article distributed under the terms of the Creative Commons Attribution-NonCommercial-ShareAlike 3.0 License, which allows others to remix, tweak, and build upon the work non-commercially, as long as the author is credited and the new creations are licensed under the identical terms

This article was originally published in a special issue, "Trends in Therapeutic Management of Various Clinical Conditions II" Indian J Pharm Sci 2021:83(2)Spl issue;1-6 